\title{
L'enseignement de la musique arabo-andalouse à
} Fès

\section{Marc Loopuyt}

\section{OpenEdition}

\section{Journals}

Édition électronique

URL : http://journals.openedition.org/ethnomusicologie/2295

ISSN : 2235-7688

\section{Éditeur}

ADEM - Ateliers d'ethnomusicologie

Édition imprimée

Date de publication : 1 janvier 1988

Pagination : 39-45

ISBN : 2-8257-0159-9

ISSN : 1662-372X

\section{Référence électronique}

Marc Loopuyt, "L'enseignement de la musique arabo-andalouse à Fès », Cahiers d'ethnomusicologie

[En ligne], 1 | 1988, mis en ligne le 15 août 2011, consulté le 01 mai 2019. URL : http:// journals.openedition.org/ethnomusicologie/2295 


\title{
L'ENSEIGNEMENT DE LA MUSIQUE ARABO-ANDALOUSE Ã FẼS
}

\author{
Marc Loopuyt
}

Ayant été pendant deux ans élève à l'Ecole de musique de Fès, je commencerai par décrire l'enseignement tel qu'il se dispensait encore en 1973, puis j'évoquerai les transformations qu'il a subies depuis.

En 1972, l'établissement se trouve dans la médina, près du mausolée de Sidi Ahmed Chaoui : c'est une grande maison traditionnelle, appelée Dār Aḍil. Disposées autour d'un vaste patio avec fontaine, six grandes pièces abritent les cours et une septième l'administration. Cette école est rattachée au Ministère de la culture, mais son fonctionnement et son statut sont complètement différents de ceux des autres écoles de musique marocaines, organisées, elles, sur le modèle des conservatoires occidentaux.

Dans cette institution séculaire, tous les enseignants sont des musiciens traditionnels de haut niveau, appartenant soit à la lignée du maître El Brihi, soit à celle du maître El Mtahiri. La plupart font partie de l'orchestre du maître Abdelkrim Raïs, qui assume aussi la fonction de directeur.

La pédagogie appliquée est bien sûr celle à laquelle ils ont été eux-mêmes soumis ; leur solide formation relève exclusivement de la tradition orale, avec un accent méthodique particulier sur le développement de la mémoire, l'apprentissage du rythme et la conjonction subtile de ces deux domaines.

Au début, une règle absolue est à observer : pas de pratique d'instruments mélodiques avant la troisième année; ainsi, la suprématie de la voix sur les instruments est affirmée d'emblée.

Le choix de la première nouba (suite musicale arabo-andalouse) abordée est très significatif de la démarche musulmane sacralisante: c'est la suite Ramal Maya, dont le thème poétique est un panégyrique du Prophète Mohammed. Cette évocation de la personne du prophète de l'Islam au début de l'étude est à mettre en rapport avec sa fonction d'Envoyé : le Coran est descendu sur lui, et il l'a transmis oralement aux premiers musulmans. Ce n'est que longtemps après l'achèvement de la révélation, alors que ses compagnons savaient par cœur l'ensemble des sourates, que s'est fait sentir la nécessité de les fixer par écrit pour éviter tout danger de déviation. Le fondement de la civilisation islamique explique la prédominance de la fonction auditive chez les Arabes; c'est le seul mode de transmission logique dans le monde bédouin originel où la poésie, le chant et la rhétorique concen- 
trent la quasi-totalité de l'expression artistique. Dans l'optique traditionnelle, cette association à la "lumière mohammedienne» (nür muhammadiya) est opérationnelle, car l'Envoyé représente la perfection de l'homme réalisé. Or, tout prétendant à un savoir et, en particulier, tout apprenti visant l'acquisition d'un métier traditionnel, en l'occurrence celui de musicien, y aspire aussi en tant que moyen de réalisation spirituelle. Le recoupement des confréries et des corporations de la médina de Fès en est une preuve évidente.

L'enseignement musical est gratuit; les élèves viennent trois fois par semaine à raison de deux heures par séance. Le premier impératif est d'arriver à la leçon en sachant par cœur le poème proposé. En général, les élèves l'ont recopié ou appris directement d'une édition du Haik (recueil du XVIII siècle). Remarquons qu'en tant que jeunes habitants de la médina, ils sont déjà familiarisés avec les mélodies et le style instrumental, car la présence de musiciens est intimement liée à de nombreux événements de la vie sociale et familiale.

Le cours commence par une répétition collective du texte; on aborde ensuite la scansion des chants, pilier central de la méthode d'enseignement. Celle-ci se combine à un système très efficace de frappes de la main droite sur la jambe droite, l'élève étant assis. Le coup fort, main ouverte, correspond au temps fort (dum); le coup effectué main fermée, doigts joints, au temps faible (tak), et en cas de longs silences, la main pratique une façila («virgule») qui consiste en un mouvement de revers vers l'extérieur.

Moins explicite que son équivalent turc l'usul, ce système appelé 'iqac fournit l'ossature de chaque rythme, il propose la perception globale de sa spécificité, sans aucune indication quantitative. Une fois assimilé, le 'iqa ${ }^{c}$ est utilisé pour scander la mélodie dont l'apprentissage se fait progressivement, vers par vers.

Ainsi, une mémorisation très efficace est assurée par la combinaison méthodique du 'iqa ${ }^{c}$, de la mélodie et du mètre poétique de la touchih (forme poétique arabo-andalouse), associés à deux autres éléments, dont l'incidence varie avec la sensibilité de l'élève : les images poétiques et les allusions philosophiques, fréquentes dans les textes chantés. A ce stade, l'orientation de la méthode d'apprentissage est donnée implicitement.

Remarquons que les enfants acquièrent dès leur plus jeune âge tout un substrat d'éducation traditionnelle à travers la récitation coranique, qui constitue pour eux une sollicitation et un développement considérable de la mémoire rythmique. Le mètre poétique du Coran étant non mesuré, son rythme subtil requiert une concentration de chaque instant. Au msìd (école primaire coranique), la méthode est collective et consiste en une répétition inlassable, très rythmée et à haute voix, accompagnée d'un balancement continuel du corps. En cours d'apprentissage, chaque enfant mémorise pour lui-même le passage de la sourate étudiée en le criant à tue-tête pendant que, dans la cacophonie générale, le fqĭh (maître d'école coranique) répète et corrige les fautes de prononciation. Ce n'est qu'une fois su par tous que le passage en question sera repris à l'unisson. Ce type d'apprentissage privilégiant la concentration se révèle d'une telle efficacité que la plupart des anciens élèves continuent à l'appliquer chaque fois qu'ils ont quelque chose à 
mémoriser. En fait, bien souvent avant l'adolescence, de jeunes garçons connaissent l'intégralité du Coran, ce qui semblerait inconcevable par le simple recours à la mémoire telle que nous l'utilisons aujourd'hui en Occident.

Cette évocation engage à penser que l'expression "par cœur» peut aussi être comprise dans le sens de "par le moyen du cœur». Elle est à envisager selon la perspective unanime et universelle des traditions qui situent dans cet organe le siège de la connaissance unitive, et elle convient parfaitement à une discipline qui, par définition, doit être dhikru 'llāh, "souvenir de Dieu".

Une fois que les premiers chants sont en voie d'assimilation, une séance sur trois est consacrée à l'exécution du rythme sur la darbuka (tambourcalice). Ainsi, le bsit, premier rythme du cycle de la nouba et de l'apprentissage, sera inculqué de la façon décrite plus haut. Habitués dès leur plus jeune âge à la frappe des $t a^{c} \overline{a r j}$ (petits tambours de terre cuite), les élèves n'y éprouvent aucune difficulté. Au contraire, et ici on mesure l'importance de l'imprégnation musicale, le maître doit plutôt réfréner la tendance des élèves à l'ornementation $(z w \bar{a} q)$. En effet, et c'est un autre trait de l'éducation, l'exubérance de leur tempérament porte souvent les musiciens débutants à une

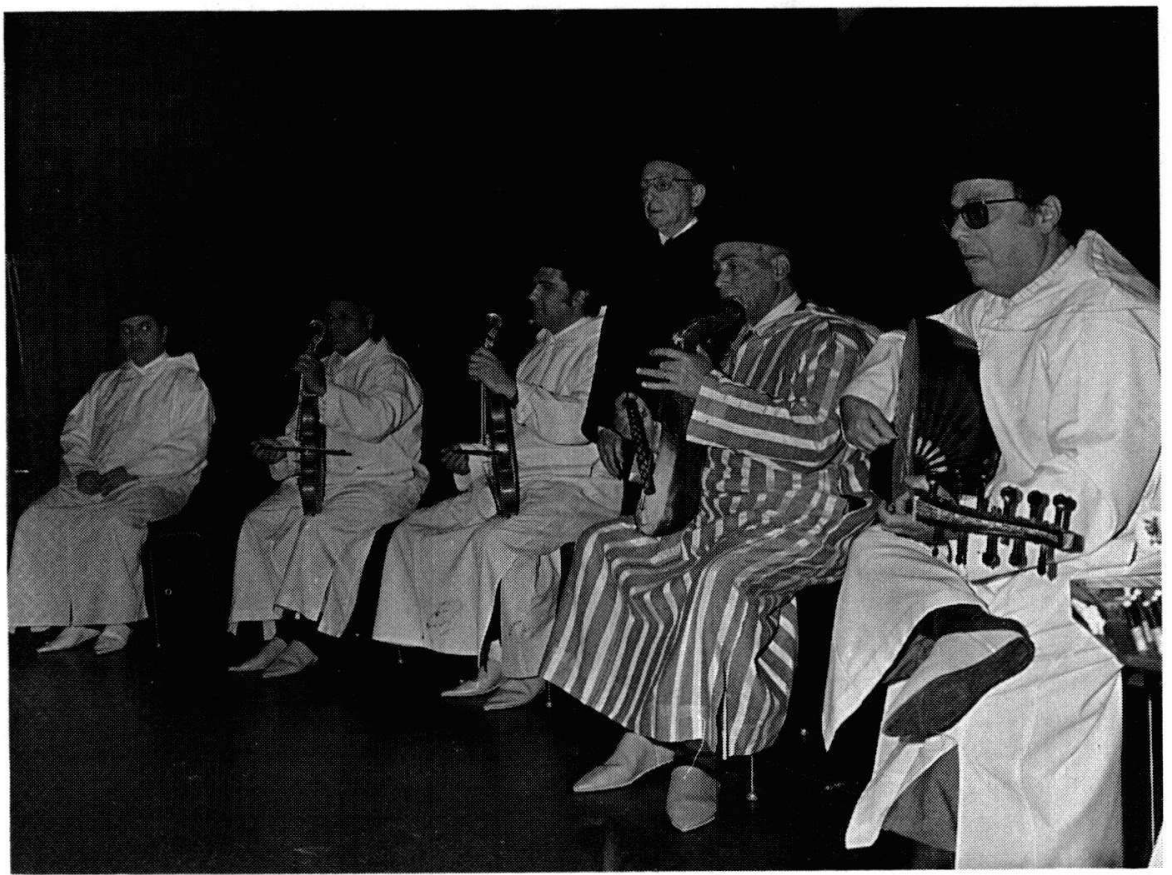

Abdelkrim Raïs (deuxième depuis la droite) et son ensemble. Debout: Driss Ben Jeloun (photo: Habib H. Touma). 


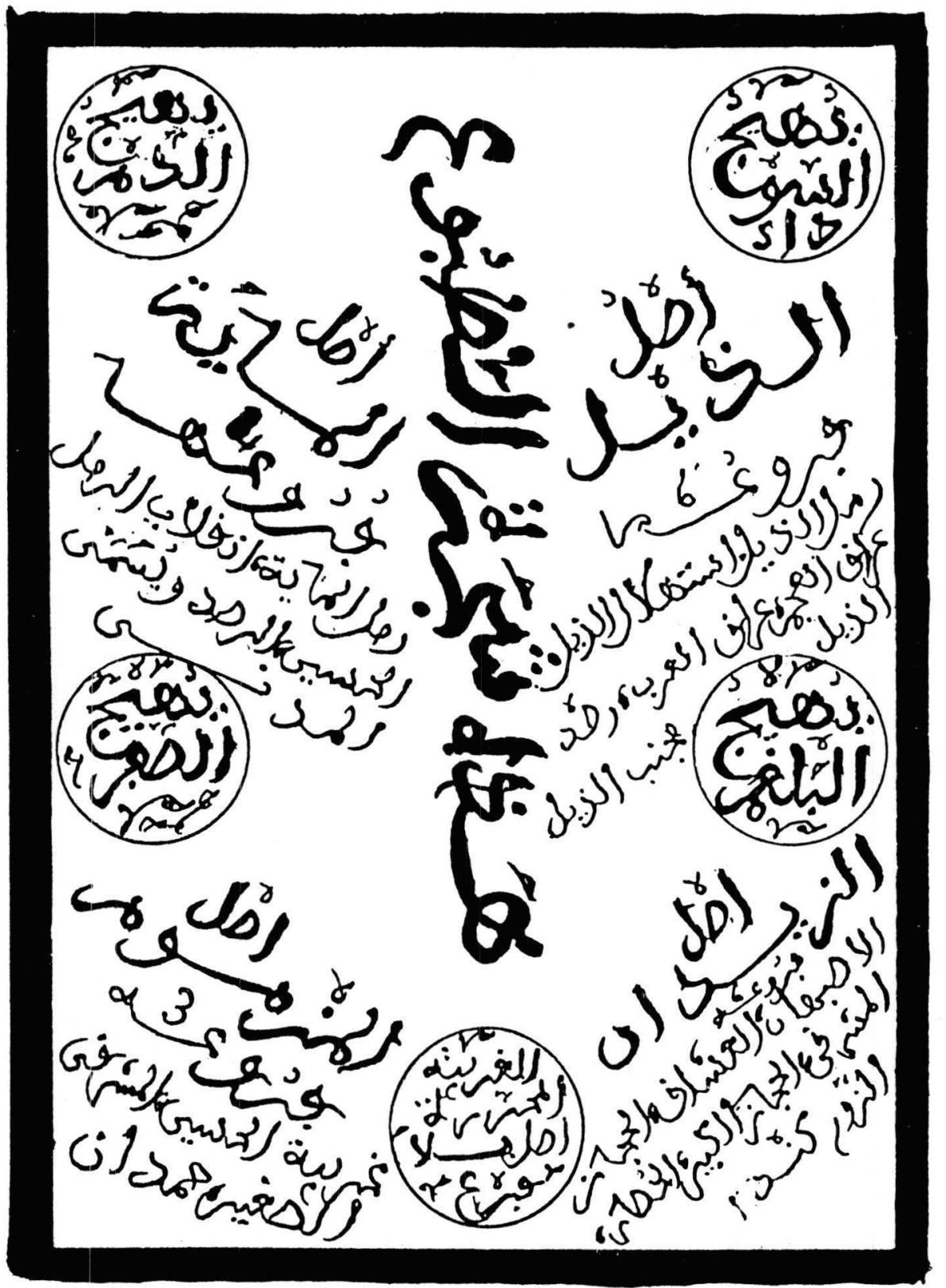

L'arbre des modes de la musique arabo-andalouse. Fès, Maroc. 
prolixité que les anciens proscrivent sévèrement, Cette rigueur de l'enseignement ne s'estompe que vers la fin du cycle: tout le monde sait bien que sans un minimum d'arabesques, la spécificité du langage musical araboandalou disparaîtrait. Les musiciens médiévistes contemporains cherchant avec plus ou moins de bonheur - les sources de leur propre tradition dans la musique arabo-andalouse en savent quelque chose.

Vient ensuite la synchronisation entre la voix et la darbuka, laquelle, après assimilation du 'iqa ${ }^{c}$, ne pose aucun problème. La méthode se poursuit selon le développement de la nouba à raison d'un chant par formule rythmique. Neuf de ces chants sont ainsi assimilés successivement, présentant un condensé minimal de la nouba Ramal Maya.

Après ce premier cycle, on aborde les neuf compositions de la nouba Isbihan de la même manière. L'ensemble constitute le programme de la première année.

La méthode se poursuit l'année suivante par l'acquisition d'autres pièces, complétées par l'apprentissage du tār, petit tambour-sur-cadre à cymbalettes, considéré comme l'instrument de percussion noble dans el 'alä, la musique arabo-andalouse vocale et instrumentale. Sa technique est complexe et son jeu subtil, car une très grande souplesse du poignet gauche est requise pour faire vibrer les cymbalettes sans jamais les toucher directement.

La troisième année, l'élève choisit un instrument: soit le ${ }^{c} u d$, soit le violon et, une fois par semaine, une séance collective réunit quinze à vingt élèves luthistes ou violonistes, qui tentent de reproduire sur leur instrument une des pièces vocales qu'ils connaissent par cœur depuis un ou deux ans. Chacun choisit sa pièce favorite et la joue dans un vacarme tel que la concentration de chacun est mise à rude épreuve. Les indications techniques se réduisent au strict minimum, mais très vite, l'assimilation préalable du répertoire vocal permet aux élèves de traduire la trame mélodique des airs. Ensuite, leur sens rythmique leur permettra de trouver d'instinct les bons coups de plectre ou d'archet. Il faudra près d'un an au futur muscien pour que, de plus en plus livré à lui-même, il puisse aborder l'expression instrumentale proprement dite.

Les élèves d'extraction modeste ne possèdent pas d'instruments, et ceux-ci leur sont prêtés par l'école, mais ils ne peuvent en principe pas en sortir. Aussi, en dehors des cours réguliers, les élèves reviennent-ils à Dār Aḍ̂il pour s'exercer dans le calme. C'est pour cette raison par exemple que les gauchers ne peuvent pas inverser les cordes de leur instrument, et qu'ils apprennent avec les graves en bas et les aigus en haut. Ils ont d'ailleurs la réputation d'être d'excellents instrumentistes et, lorsqu'ils acquièrent leur propre instrument, ils continuent à monter les cordes de cette façon.

A partir de la quatrième année, les élèves les plus déterminés vont entreprendre une étape nouvelle et très importante de leur apprentissage : ils vont progressivement s'intégrer à de petits orchestres semi-professionnels en tant que percutionnistes occasionnels. C'est à l'occasion des fêtes, au cours d'engagements dans les maisons privées, qu'ils vont élargir leur technique et leur répertoire, non comme ils l'ont fait à l'école, mais "sur le tas", sans aucune décomposition pédagogique des mouvements. Toujours par la conjonction du 
chant et du rythme, ils poursuivent ainsi le long chemin de l'apprentissage. C'est pour eux l'occasion d'approcher des artistes de tous niveaux et de tisser de nouveaux liens d'intimité artistique, jusqu'à être éventuellement sollicités par leurs maîtres de Dār Aḍ̂il.

C'est aussi dans ces circonstances, et non à l'école où il n'y a pas de classe d'orchestre, que s'apprend d'oreille la manière d'orner les compositions propres à chaque instrument. A ce stade déjà, le tarāb, ou «ivresse» artistique, propre à la musique arabo-andalouse, est un adjuvant puissant et nécessaire qui va contribuer, sur la base des solides fondements reçus préalablement, à cimenter l'édifice du savoir.

Généralement, les élèves qui parviennent au terme de dix années d'apprentissage sont déjà des semi-professionels, car, pour bon nombre d'entre eux, l'aspect économique du métier constitue en soi une motivation importante. La plupart sont issus de familles de modestes artisans de la médina, de foyers de bienfaisance ou d'orphelinats, aussi n'est-il jamais trop tôt pour commencer à gagner sa vie, même modestement.

Tels étaient jusqu'en 1973 les grands traits de l'éducation musicale à Dār Aḍil. Dès 1974, la création d'une classe de solfège révéla clairement une intention de modifier les bases de l'enseignement en accord avec les transformations de la vie marocaine. Le problème qui se pose ici réside dans les raisons de cette innovation : quelle utilisation entend-on faire du solfège ? S'il s'agit de noter les compositions apprises pour être sûr de ne pas les oublier, pourquoi pas ; mais s'il s'agit de remplacer l'enseignement de bouche à oreille, ou plutôt de "cœur à cœur", par une pédagogie figée dans l'écriture, cela risque de rompre les précieuses chaînes du savoir intégral. Ceci d'autant plus que Dār Aḍ̂il a entre-temps été fermé et que l'établissement a déménagé dans la ville moderne. Ainsi la musique a dû se plier aux exigences de normalisation qui a déjà poussé de nombreux notables hors de la médina. Il est à craindre qu'une telle tendance simplificatrice - l'introduction de l'écriture musicale - ne fasse jouer activement les préjugés modernistes pour mettre à mal ce système de transmission artistique.

Evidemment, il est facile de mettre en exergue, sous de fallacieux prétextes de rendement quantitatif, les inévitables imperfections du système, notamment la tendance thésaurisante de certains maîtres, qui en arrivent à emporter des compositions entières du répertoire dans la tombe. Quoi qu'il en soit, ce qu'a été Dār Aḍ̂il et ce qu'est la nouvelle école de Fès n'empêchent pas d'autres formes de transmission de fonctionner discrètement: transmission de maître à disciple avancé au sein d'un orchestre, transmission à l'intérieur des familles ou entre amis. C'est notamment le cas du rbāb (vièle à archet), emblème du chef d'orchestre dans la musique arabo-andalouse, dont le jeu ne s'enseigne jamais explicitement, mais qui est recueilli avec la dignité correspondante par le plus qualifié des proches d'un maître défunt.

Le plus grand risque est que le nouveau mode de transmission ne sache pas prendre en compte la dimension spirituelle inhérente à la musique araboandalouse. En effet, comme l'a magistralement exposé Titus Burckhardt (1987 : 227), la notion traditionnelle d'art (fann) implique celles de savoir-faire artisanal $\left(\operatorname{csin}^{c} a\right)$ et de science $\left({ }^{c} i l m\right)$, elles-mêmes liées à celle de sagesse 
(hikmat), qui est la relation des choses à leur principe universel. Dans la musique arabo-andalouse, l'aspect $\operatorname{çin}^{c} a$ correspond à la maîtrise formelle impliquant la mémorisation, le contrôle des timbres, l'ornementation et la paraphrase, vocale ou instrumentale; l'aspect ${ }^{c}$ ilm est la science du nombre dans le mètre musical et poétique et la connaissance ( $\left.{ }^{c} i r f a ̄ n\right)$ de l'arbre des modes dans ses implications anthropologiques et cosmologiques; quant à l'aspect hikmat, il est nécessairement relatif au sama ${ }^{c}$, à l'écoute recueillie par l'oreille du cœur, qui doit pouvoir assimiler toute mélodie à son mode $\left(t a b a^{\mathcal{C}}\right)$, à son archétype et, en dernière analyse, au son primordial. Telle est l'ampleur de l'héritage de Ziryāb qui, virtuellement du moins, est toujours accessible, comme l'indique un des noms de la musique arabo-andalouse: tarāb ${ }^{c}$ andalusi. Venant de la racine $T R B$, qui désigne une «légèreté qui atteint l'âme sous l'effet d'une forte émotion ", cette expression évoque la science cathartique de l'âme qu'on trouve dans le soufisme, ce que confirme la teneur de nombreux poèmes chantés.

Il est évident qu'une institution calquée sur les conservatoires européens ne peut prendre en compte cette dimension du savoir que si elle sait confier son enseignement à des maîtres en la matière. On pourrait imaginer une redistribution des tâches de transmission, mais la difficulté réside dans le complexe de supériorité des musiciens formés à l'écriture musicale. Gageons que des esprits clairvoyants sauront justement relever ce défi, et qu'ainsi pourront perdurer et même reverdir les richesses de leur héritage.

\section{Bibliographie}

BURCKHARDT Titus

1987 Mirror of the intellect. Essays on traditional science and sacred art. Cambridge : Quinta Essentia. 